\section{Dave Singh}

North West Lung Research Centre South Manchester University Hospitals Trust Manchester, UK M23 9LT

\section{The benefits of combined treatment with corticosteroids and long- acting beta agonists}

Guidelines for the treatment of COPD recommend that long-acting beta agonists (LABA) are used initially to maximize lung function and relieve symptoms in patients with mild to moderate disease who are being treated with short-acting bronchodilators (www.goldcopd.com). Inhaled corticosteroid (ICS) therapy reduces exacerbation rates in patients with more severe disease (Burge et al 2000), and so guidelines recommend that these drugs are used for this purpose primarily in patients with $\mathrm{FEV}_{1}<50 \%$ predicted. ICS and LABA can be co-administered using combination inhalers, which give practical advantages as well as increasing compliance and maximizing the chance of synergistic interactions between the monocomponents. There is evidence that the combined LABA/ICS inhalers salmeterol/fluticasone propionate (Sal/FP) (Calverley et al 2003a) and formoterol/budesonide (F/Bud) (Calverley et al 2003b; Szafranski et al 2003) reduce exacerbation rates. Our current treatment strategies using LABA and ICS therefore have different goals (optimizing lung function, symptom relief, and exacerbation reduction), and are stratified by lung function.

In the current issue, Calverley et al ( $p$ 209-18) have re-analyzed data from the TRISTAN study which compared the effects of Sal/FP, FP alone, Sal alone, and placebo over 1 year on lung function, exacerbation rates, and health status in order to evaluate whether the pre-treatment $\mathrm{FEV}_{1}$ influenced these outcome measures. The $\mathrm{FEV}_{1}$ data were categorized using the arbitary cut off value of $50 \%$ predicted, but also analyzed as a continuous variable. The authors note the limitations of a post hoc analysis, and that the study was primarily designed to evaluate the difference in $\mathrm{FEV}_{1}$ between Sal/FP and placebo, rather than differences between the active treatments. Nevertheless, this paper provides a wealth of important data on the effects of different treatments on different endpoints across a range of disease severity stratified by $\mathrm{FEV}_{1}$.

$\mathrm{Sal} / \mathrm{FP}$ significantly improved the pre-bronchodilator $\mathrm{FEV}_{1}$ at all disease severities compared with FP, Sal, and placebo. Interestingly, the benefit of Sal/FP compared with its monocomponents Sal and FP appeared to be synergistic in patients with more severe disease, but additive in patients with milder disease. It is known that LABA and ICS have synergistic interactions in vitro (Johnson 2004), and these clinical trial data suggest that the degree of synergy for lung function depends on disease severity. FP alone had no significant effect in patients with the most severe disease. It could be argued that Sal "unlocks" corticosteroid insensitivity in these patients, leading to a true synergistic effect. Although it is known that Sal/FP reduces airway inflammation in bronchial biopsies compared with placebo (Barnes et al 2006), no similar data compare Sal/FP with its monocomponents. Such a study, across a range of disease severities, would facilitate our understanding of the molecular interactions between Sal and FP in vivo.

Sal/FP, Sal, and FP all significantly reduced exacerbation rates compared to placebo, but only in patients with $\mathrm{FEV}_{1}<50 \%$ predicted. This lung function cut-off value appears to be useful for predicting which patients are most likely to benefit from Sal/FP combination therapy to reduce exacerbations. However, the effect of $\mathrm{Sal} / \mathrm{FP}$ was similar to that of the monocomponents, raising the question of whether patients can be treated with moncomponents alone for exacerbation reduction. 
However, there is an advantage of combination therapy over the monocomponents for lung function and health status. The optimum treatment strategy for COPD must be to take a global view of the disease, encompassing a number of clinical endpoints. With this in mind, the TRISTAN reanalysis indicates that the advantage of combination therapy with Sal/FP over the monocomponents is most apparent for lung function and health status. Indeed, the observed synergistic effect on lung function between Sal and FP in patients with more severe disease, and the lack of significant effect of FP on lung function in these patients, strongly suggests that FP is most effectively prescribed with Sal in these patients.

The review article by Chung (p 235-42) in the current issue raises the issue of the dose of Sal/FP used in clinical practice, as we have most information using a daily FP dose of 1000ìg, but dose-response effects on different therapeutic endpoints may exist. Furthermore, while there is no evidence that the Sal/FP combination is better than its monocomponents for reducing exacerbations, there are different data for the F/Bud combination, which is more effective than its monocomponents (Calverley et al 2003b; Szafranski et al 2003). The differences between F/Bud study results and the TRISTAN data may be attributed to different designs, inclusion criteria, and exacerbation definitions. However, the ICS and LABA components of Sal/FP and F/ Bud have different pharmacological properties, and are administered by different inhaler devices, so it is possible that there may be intrinsic differences in their clinical effect profiles.

Mannino and Kiri (p 219-33) review in the current issue strategies for changing the burden of COPD mortality, including the use of ICS and combination therapies. They highlight the paucity of data from randomized controlled trials for a benefit of ICS on survival, except for one metaanalysis (Sin et al 2005). Non-randomized, observational cohorts may suffer from immortal time bias, which occurs when a subject dies before treatment is initiated and so is included in the control group. In a study free of immortal time bias, there is a mortality benefit for ICS (Kiri et al 2005), although there are no similar data for combination therapies. There is clearly a need for more data on survival with combination therapies from prospective, randomized trials. The full results of the TORCH (Towards a Revolution in COPD Health) study, prospectively comparing the effects of Sal/FP, Sal, FP, and placebo on mortality, exacerbations, lung function, and health status over 3 years are eagerly awaited.
COPD is a heterogenous disease, and the phenotypes of disease that may predict disease prognosis or response to therapy need better definition. The therapeutic response to corticosteroid therapy is COPD is variable, with the greatest benefit observed in patients with sputum eosinophilia (Brightling et al 2000, 2005). Identification of the characteristics of patients most likely to benefit from combination therapy with ICS/LABA would allow these drugs to be used rationally and cost effectively. This will be a complex task, as combination therapy appears to have varying effects on different clinical endpoints. It is therefore possible that the phenotype of patients who respond to combination therapy may vary with the endpoint studied, eg, the phenotype of "lung function responders" may differ from those who show reduced exacerbations. We need detailed characterization in clinical trials to understand fully the phenotype of responders and nonresponders.

\section{References}

Barnes NC, Qiu YS, Pavord ID, et al. 2006. Antiinflammatory effects of salmeterol/fluticasone propionate in chronic obstructive lung disease. Am J Respir Crit Care Med, 173:736-43.

Brightling CE, McKenna S, Hargadon B, et al. 2005. Sputum eosinophilia and the short term response to inhaled mometasone in chronic obstructive pulmonary disease. Thorax, 60:193-8.

Brightling CE, Monteiro W, Ward R et al. 2000. Sputum eosinophilia and short-term response to prednisolone in chronic obstructive pulmonary disease: a randomised controlled trial. Lancet, 356:1480-5

Burge PS, Calverley PM, Jones PW, et al. 2000 Randomised, double blind, placebo controlled study of fluticasone propionate in patients with moderate to severe chronic obstructive pulmonary disease: the ISOLDE trial. BMJ, 320:1297-303

Calverley PM, Pauwels R, Vestbo J, et al. 2003a. Combined salmeterol and fluticasone in the treatment of chronic obstructive pulmonary disease: a randomised controlled trial. Lancet, 361:449-56.

Calverley PM, Boonsawat W, Cseke Z et al 2003b. Maintenance therapy with budesonide and formoterol in chronic obstructive pulmonary disease. Eur Respir J. 2003;22:912-19

Johnson M. 2004. Interactions between corticosteroids and beta2-agonists in asthma and chronic obstructive pulmonary disease. Proc Am Thorac Soc, 1:200-6.

Kiri VA, Pride NB, Soriano JB, et al. 2005. Inhaled corticosteroids in chronic obstructive pulmonary disease: results from two observational designs free of immortal time bias. Am J Respir Crit Care Med, 172:460-4.

Sin DD, Wu L, Anderson JA et al. 2005. Inhaled corticosteroids and mortality in chronic obstructive pulmonary disease. Thorax, 60:9927.

Szafranski W, Cukier A, Ramirez A et al. 2003. Efficacy and safety of budesonide/formoterol in the management of chronic obstructive pulmonary disease. Eur Respir J, 21:74-81. 